\title{
Bare Neutron Counter and Neutron Monitor Response to Cosmic Rays during a 1995 Latitude Survey
}

\author{
W. Nuntiyakul ${ }^{* a, b}$, A. Sáiz ${ }^{c}$, D. Ruffolo ${ }^{c}$, P.-S. Mangeard ${ }^{d, e}$, P. Evenson ${ }^{e}$, J. W. Bieber ${ }^{e}$, \\ J. Clem $^{e}$, R. Pyle ${ }^{f}$, M. L. Duldig ${ }^{g}$, J. E. Humble ${ }^{g}$ \\ ${ }^{a}$ Department of Physics and Materials Science, Faculty of Science, Chiang Mai University, \\ Chiang Mai 50200, Thailand \\ ${ }^{b}$ Research Center in Physics and Astronomy, Faculty of Science, Chiang Mai University, Chiang \\ Mai 50200, Thailand \\ ${ }^{c}$ Department of Physics, Faculty of Science, Mahidol University, Bangkok 10400, Thailand \\ ${ }^{d}$ National Astronomical Research Institute of Thailand (NARIT), Chiang Mai 50180, Thailand \\ ${ }^{e}$ Department of Physics and Astronomy, University of Delaware, Newark, DE 19716, USA \\ ${ }^{f}$ Pyle Consulting Group, Inc., St. Charles, IL 60174, USA \\ ${ }^{g}$ School of Natural Sciences, University of Tasmania, Hobart, Tasmania 7001, Australia \\ E-mail: waraporn.n@cmu.ac.th, alejandro.sai@mahidol.ac.th, \\ david.ruf@mahidol.ac.th,psmangeardegmail.com,evenson@udel.edu, \\ jwbieber@bartol. udel.edu,clem@bartol. udel.edu, RogerPyle@gmail.com, \\ Marc.Duldig@utas. edu . au, John. Humble@utas . edu . au
}

Neutron monitors of standard design (IGY or NM64) are employed world-wide to study variations in the flux of galactic cosmic rays and solar energetic particles in the $\mathrm{GeV}$ range. The design minimizes detector response to neutrons below $\sim 10 \mathrm{MeV}$ produced by cosmic ray interactions in the ambient medium. Increasingly, however, such neutrons are of interest as a means of obtaining spectral information on cosmic rays, for studies of soil moisture, and for nuclear threat detection. Bare neutron counters, a type of lead-free neutron monitor, can detect such neutrons, but comparatively little work has been done to characterize the dependence of their count rate on cutoff rigidity. We analyze data from three bare neutron counters operated on a ship together with a 3-tube NM64 monitor from November, 1995 to February, 1996 over a wide range of magnetic latitude, i.e., a latitude survey. The bare counter design used foamed-in-place polyurethane insulation to keep the temperature uniform and to some extent moderate high-energy neutrons. When the ship was near land, the bare/NM64 count rate ratio was dramatically higher. Considering only data from open sea, the bare and NM64 pressure coefficients are not significantly different. We determine the response function of these bare counters, which is weighted to Galactic cosmic rays of lower energy than the NM64. This measurement of the response function may improve determination of the spectral index of solar energetic particles and Galactic cosmic rays from a comparison of bare and NM64 count rates.

36th International Cosmic Ray Conference -ICRC2019-

July 24th - August 1st, 2019

Madison, WI, U.S.A.

* Speaker. 


\section{Introduction}

Neutron monitors are ground-level detectors of cosmic-ray-induced atmospheric secondary particles that respond mostly to secondary neutrons [12]. Neutron monitors of the standard IGY and NM64 designs are optimized so that their count rates provide a precise and reliable measurement of the GeV-range cosmic-ray flux [8]. In particular, a neutron monitor at a given location is most sensitive to cosmic rays within a range in rigidity (momentum per charge, expressed in GV) somewhat above the local geomagnetic cutoff, which varies from near $0 \mathrm{GV}$ at Earth's polar regions to $\sim 17 \mathrm{GV}$ at some parts of the geomagnetic equator, and above the atmospheric cutoff of $\gtrsim 1 \mathrm{GV}$ needed to generate atmospheric secondary particles that can be recorded at ground level (see the response functions determined by [9]). A standard neutron monitor includes a lead producer to increase the response to high energy primary cosmic rays. Standard neutron monitors are operated at more than 40 locations worldwide, taking advantage of the varying geomagnetic cutoff, and are widely used to monitor Earth's radiation environment due to relativistic solar energetic particles and galactic cosmic rays and their variations associated with solar activity, and for applications concerning space weather and space radiation.

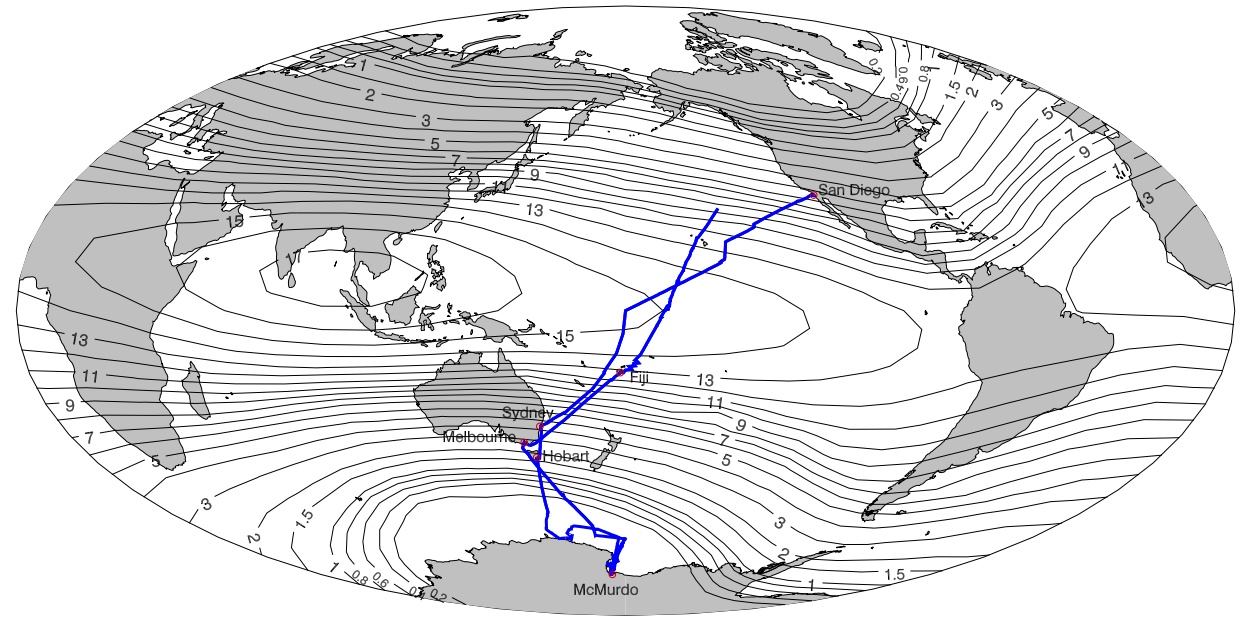

Figure 1: Track of the USCGC Polar Star for the 1995-1996 survey, superimposed on contours of vertical cutoff rigidity. Numbers give vertical cutoff rigidity in GV. This survey carried a standard neutron monitor (3NM64) and three bare neutron detectors, permitting a direct comparison of their response to primary cosmic rays.

Such observations can be complemented by measurements using bare neutron counters that lack the reflector and lead producer of standard neutron monitors, making them also sensitive to atmospheric neutrons of $\leq 10 \mathrm{MeV}$. Such detectors are widely used to detect soil moisture [15], for nuclear threat detection [7], and to obtain spectral information on solar energetic particles.

From a fixed location, a standard neutron monitor (NM64) count rate alone cannot be used to measure the energy spectrum of cosmic rays. However, since a bare counter has a different energy response from an NM64, operating them together provides some knowledge of the spectrum [e.g., [1, 3]] while avoiding the systematic errors of comparing count rates from different locations [figure 2 of [11]]. The accuracy of this method requires knowing how the energy responses of the different types of detector are related. 
In this work we focus on developing optimal methods for extracting the response function of three bare counters that were operated aboard the U.S. Coast Guard icebreaker Polar Star along the route illustrated in Figure 1. This voyage was one of a series of latitude surveys in which the magnetic field of the Earth was used as a spectrometer to explore the time dependence of the spectrum of cosmic rays striking the atmosphere [9]. All of these surveys carried a NM64 neutron monitor with 3 counter tubes, i.e., a 3NM64, and in addition the survey from San Diego, USA to McMurdo, Antarctica and back during a voyage of 4 months from 6 November 1995 to 20 March 1996 carried three bare detectors. The key results have also been presented by [10].

\section{Methodology}

\subsection{Latitude Surveys and Response Functions}

The count rate $N(t)$ of a ground based detector resulting from the impact of cosmic rays at the top of the atmosphere is described by

$$
N(\Theta, \Phi, h, t)=\int_{0}^{\infty}\left[\sum_{i} J_{i}(P, t) Y_{i}(P, h)\right] T(P, \Theta, \Phi, t) \mathrm{d} P,
$$

where $J_{i}$ is the Galactic cosmic ray spectrum for primary cosmic rays of particle type $i$ near Earth expressed as a function of rigidity $(P)$ and time $(t)$. The yield function $Y_{i}$ for primary particles of type $i$, a function of rigidity $(P)$ and atmospheric depth $(h)$, is the actual relationship between the cosmic ray flux at the top of atmosphere and the observed count rate from the detectors. The transmission function $T_{i}$ describes the transmission through the magnetosphere of the Earth. It is a function of rigidity $(P)$, latitude $(\Theta)$ and longitude $(\Phi)$ of the detector location, and time $(t)$.

At a given latitude and longitude, the effective cutoff rigidity for transmission through Earth's magnetic field depends on the arrival direction of each primary cosmic ray described by the local zenith and azimuthal angles. However $T_{i}$ can be approximated by a step function at a single cutoff rigidity $P_{\mathrm{c}}$, that we call the apparent cutoff rigidity. With the assumption that the transmission is a step function, the count rate relation becomes

$$
N\left(P_{\mathrm{c}}, h, t\right)=\int_{P_{\mathrm{c}}}^{\infty} \sum_{i} J_{i}(P, t) Y_{i}(P, h) \mathrm{d} P .
$$

The apparent cutoff used in this work considers both vertically incident particles and obliquely incident particles [5]; this is calculated individually at one-hour intervals at the actual position of the ship with a time-dependent model of the magnetic field according to an efficient method [2].

The differential response function (DRF) is defined as the negative of the derivative of the count rate as a function of cutoff rigidity. The most common type of analysis for measuring a response function is called the Dorman analysis [6], in which corrections are first applied to the data points, then a parameterized function of $P_{\mathrm{c}}$, termed a Dorman function, is fitted to the data:

$$
N\left(P_{\mathrm{c}}\right)=N_{0}\left(1-\mathrm{e}^{-\alpha P_{\mathrm{c}}^{-\kappa}}\right),
$$

where $N_{0}, \alpha$, and $\kappa$ are unphysical parameters that provide a good representation of the integral response function $N$, which can be analytically differentiated to determine the DRF:

$$
N\left(P_{\mathrm{c}}\right)=\int_{P_{\mathrm{c}}}^{\infty} \mathrm{DRF}(P) \mathrm{d} P
$$




$$
\operatorname{DRF}(P)=N_{0} \alpha P^{-\kappa-1} \kappa \mathrm{e}^{-\alpha P^{-\kappa}} .
$$

From Eq. (2.2) and (2.4), we can obtain the DRF as the summed product of $J_{i}(P, t)$ and $Y_{i}(P, h)$ :

$$
\operatorname{DRF}(P)=-\left[\frac{\mathrm{d} N}{\mathrm{~d} P_{\mathrm{c}}}\right]_{P}=\sum_{i} J_{i}(P, t) Y_{i}(P, h) .
$$

\subsection{Instrumentation}

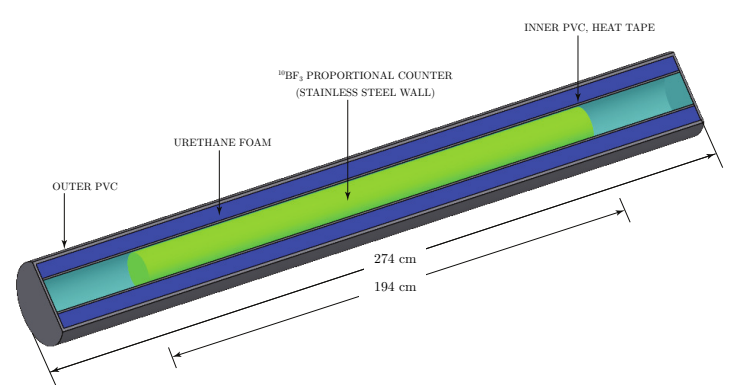

Figure 2: Rendered sketch of the "bare" neutron counter configuration used in this work. Each BP-28 neutron-sensitive proportional counter was operated inside a $20 \mathrm{~cm}$ diameter PVC pipe covered with heat tape and thermally insulated by foamed-in-place polyurethane inside a larger, $30 \mathrm{~cm}$ diameter PVC pipe.

The 1995 latitude survey employed two types of neutron detectors, three bare neutron detectors and a 3NM64 neutron monitor. The three bare counters (BCs) were installed exposed on an upper deck of the ship, whereas the 3NM64 was installed in an insulated shipping container (called the "TasVan"). The detectors are Chalk River BP-28 proportional counter cylinders filled with boron trifluoride gas (enriched in the isotope ${ }^{10} \mathrm{~B}$ ). The boron nuclei react with neutrons and undergo nuclear fission. Unlike for an NM64, there is no standard design for bare neutron counters. A rendered drawing of the detector is shown in Figure 2.

\section{Data Reduction}

\subsection{Overview}

Throughout this work all the dates during the survey will be referred to as "day of the year of 1995" (DOY1995). The 12th generation International Geomagnetic Reference Field (IGRF12) and the 1989 revision of the [13] model were used for calculating the cutoff rigidity. Numerical results for the 3NM64 in the present work differ slightly from those presented by [9] for three main reasons: (1) data intervals were selected such that they had good data in both the BCs and the 3NM64 whereas the earlier study only considered the 3NM64; (2) no corrections for short term modulation based on the McMurdo count rate were applied in this work; and (3) no normalization of the data to the McMurdo count rate was performed in this work.

\subsection{Initial Processing}

Our data were recorded with one second time resolution together with clinometer readings to allow for possible corrections due to the varying orientation of the ship in rough seas. We made histograms of the one second readings for each tube for each hour of the survey, and only used seconds with 0 to 4 counts. We eliminated the noise with one hour resolution from this stage. We deferred rejecting bad hours to a later stage of analysis. Only data with BC/NM count rate ratios within $\pm 3 \sigma$ of a reference value are selected for further analysis, as specified in [10]. 


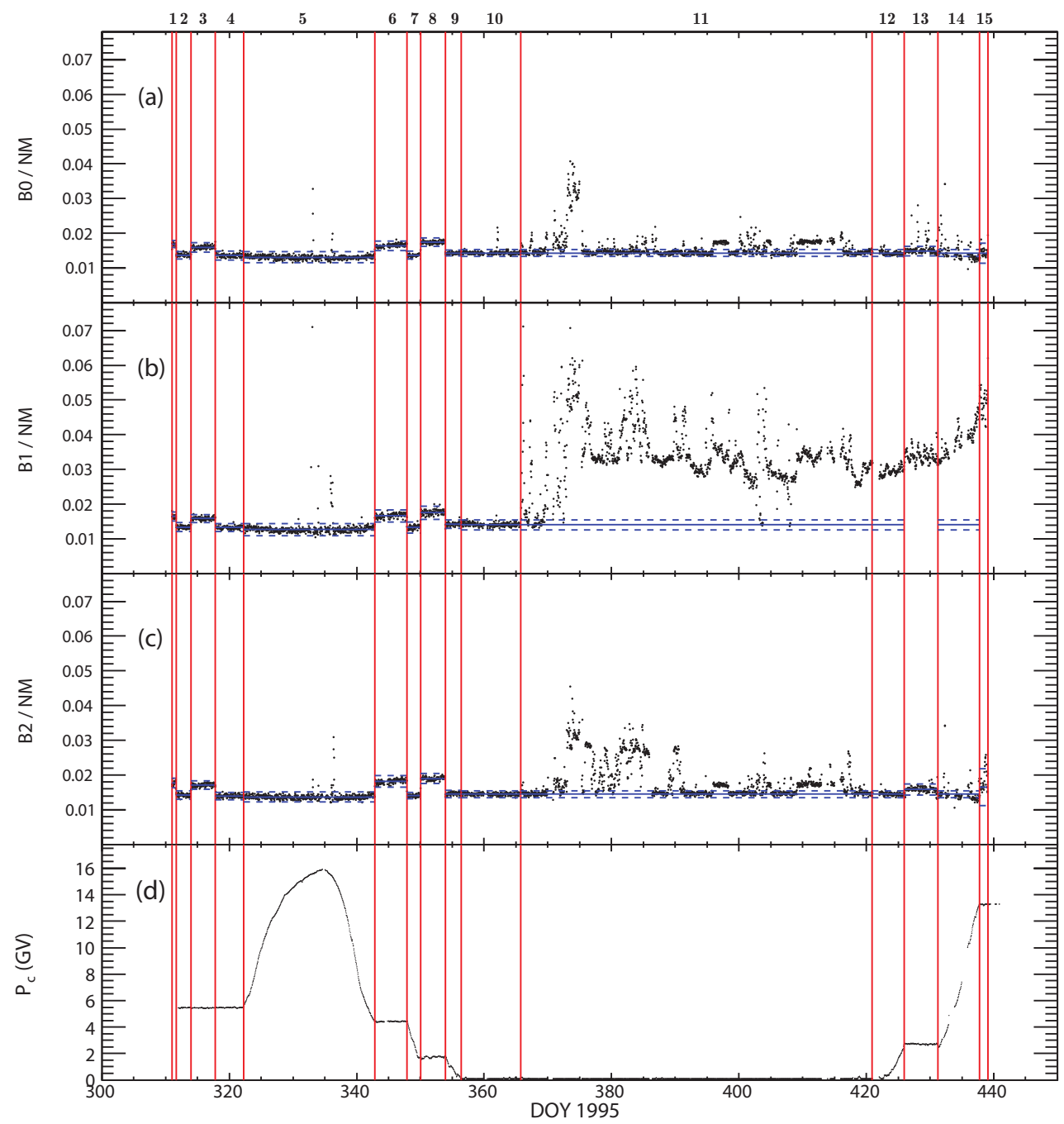

Figure 3: Summary of hourly averaged data as a function of time. (a)-(c) Ratios of individual bare detector count rates to 3NM64 count rate. Red vertical lines define 15 time periods used in the analysis. In each time period, the horizontal blue solid line shows the reference value $\left\langle B_{i} / \mathrm{NM}\right\rangle$ and blue dashed lines illustrate the $\pm 3 \sigma$ interval around the reference. (d) Apparent geomagnetic cutoff rigidity $P_{\mathrm{c}}$.

\subsection{Data Cleaning Based on Neutron Monitor Count Rate}

The selection of usable data (data cleaning) and the adjustment of bad or missing individual counter data (data correction) is performed based on BC-to-NM ratios $\left(B_{i} / \mathrm{NM}\right)$ and BC-to-BC ratios $\left(B_{i} / B_{j}\right)$. We start by choosing appropriate time periods during the 1995 survey year for which data were taken in a relative stable environment. The 15 time periods used (TP1 to TP15) are shown in Figure 3. Data cleaning is then applied separately for each time period. This division into time intervals is based on the locations near which the ship passed, i.e., San Diego, Sydney, Hobart, McMurdo, Melbourne, and Fiji, as well as on apparent step-like changes in the ratio values elsewhere. For each time period we generated histograms of the hourly $B_{i} / \mathrm{NM}$ values to characterize and remove outliers. Figure 3(a)-(c) illustrates the individual $B_{i} / \mathrm{NM}$ ratios as a function of time. The red vertical lines show the division in time periods. The blue solid horizontal 
lines illustrate the $B_{i} / \mathrm{NM}$ reference values $\left\langle B_{0} / \mathrm{NM}\right\rangle,\left\langle B_{1} / \mathrm{NM}\right\rangle$, and $\left\langle B_{2} / \mathrm{NM}\right\rangle$. In most cases, these were defined as the mean of a Gaussian fit to the ratio histogram for each time period. For three time periods, namely TP11, TP12, and TP14, we used the reference calculated during TP10 because it was noticed that the noise was too high for the center BC $\left(B_{1}\right)$. Using the TP10 ratio reference for those three problematic time periods, we avoided bias in the data that could induce further analysis errors. For the $\left\langle B_{1} / \mathrm{NM}\right\rangle$ ratio reference, during TP13 and TP15 data were also noisy but no appropriate reference based on another time period with similar environment could be identified, so those intervals have no reference available. The blue horizontal dashed lines illustrate plus and minus three standard deviations from the reference for each time period, also based on the Gaussian fit. $B_{i} / \mathrm{NM}$ data points lying outside the range defined by three standard deviations (i.e., $\pm 3 \sigma$ ) from the reference were considered to indicate bad data and the count rate $B_{i}$ was then corrected (if possible) as explained in the next section or removed before subsequent analysis.

\subsection{Data Correction Based on BC Ratios}

The rough and changing conditions on board the ships caused the response of individual BCs to occasionally change, become noisy, or even stop completely. In order to correct for these effects during the surveys, we used the inherent redundancy of the three bare detectors. The histograms of individual $\mathrm{BC}$ ratio values $\left(B_{0} / B_{1}, B_{1} / B_{2}\right.$, and $\left.B_{2} / B_{0}\right)$ were plotted for each time period (except for periods with no appropriate reference for the $B_{i} / \mathrm{NM}$ ratio, as mentioned above). For TP1TP10, reference values were calculated for these $\mathrm{BC}$ ratios based on a fit to a Gaussian, but for TP11, TP12, and TP14 we used the BC ratio reference of TP10 instead. For TP13 and TP15, the $\mathrm{BC}$ ratios were not used because the center tube had bad counts during the whole time period and there was no other appropriate reference. We defined the range of three standard deviations $( \pm 3 \sigma)$ from the reference values $\left\langle B_{i} / B_{j}\right\rangle$ to identify count rates that needed to be corrected. The reference values for the $\mathrm{BC}$ ratios were then used to correct those count rates, using the same method as explained in [9]. The count rates of the three bare tubes for each hour $\left(B_{1}, B_{2}\right.$, and $\left.B_{3}\right)$ were corrected if the $\mathrm{BC}$ ratio data were outside the $3 \sigma$ range and there was a valid $\mathrm{BC}$ ratio reference for calculating the missing data.

\subsection{Barometric Pressure Correction}

We carefully investigated the correction for variation in barometric pressure with the data from the 1995 survey. We also calculated pressure coefficients for the ratios of the summed count rate of the three BCs (3BC) to that of the 3NM64. Since there is no evidence for a major difference in coefficients from the survey data alone, in the present analysis we have corrected both $3 \mathrm{BC}$ and 3NM64 with the well-determined coefficient from the 13-year data set for the 3NM64 [9].

We also extracted coefficients from historical data for two different land-based BC configurations and the $3 \times 1$ NM64 neutron monitor at South Pole and for BCs and an 18NM64 at Doi Inthanon, Thailand. In addition, we set up BCs at Newark, Delaware (three spare BP-28 counters) and McMurdo (one BP-28 removed from the NM64) to investigate this issue. In general, there is no specific pattern with altitude or cutoff; rather the data provide an additional caution regarding the environmental sensitivity of bare detectors. In other words, the observed pressure coefficient of the shipborne BC to NM64 count rate ratio is consistent with zero, given the uncertainties. 


\subsection{Port Effects}

The extreme sensitivity of bare detectors to the environment is clearly illustrated in Figure 4, which shows the ratio of the $\mathrm{BC}$ to NM count rate after all of the corrections discussed above have been applied. Substantial increases in the ratio occur whenever the ship is in port, as was also found by [14]. This can be explained by a higher density of albedo neutrons near solid ground compared with open water, and the fact that neutron detectors lacking a reflector (such as the BCs) are much more sensitive to these environmental neutrons than the standard NM64. We therefore classified the data carefully so as to be certain that the results we report are representative of the open ocean.

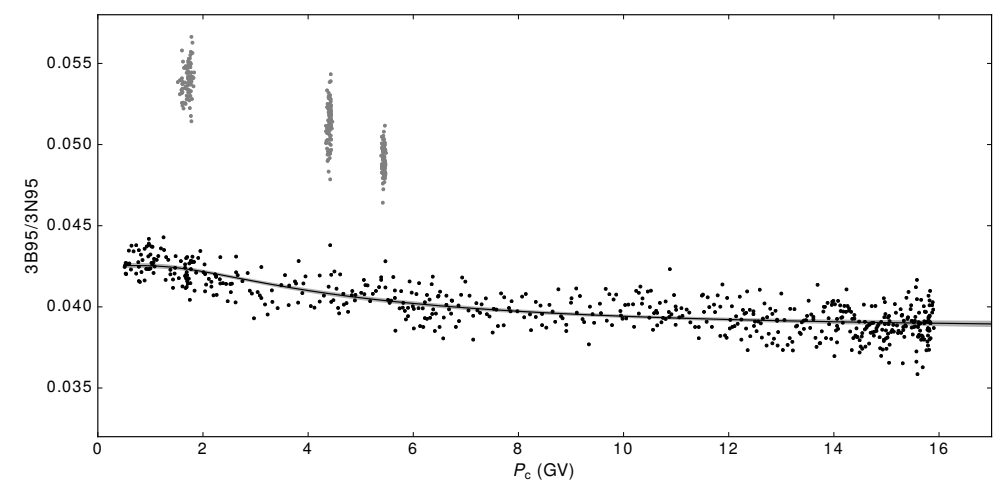

Figure 4: BC/NM ratio in 1995 as a function of apparent geomagnetic cutoff rigidity $P_{\mathrm{c}}$. Black dots: ship in open sea. Gray dots: port calls at Hobart $\left(P_{\mathrm{c}}=1.71 \mathrm{GV}\right)$, Sydney $\left(P_{\mathrm{c}}=4.41 \mathrm{GV}\right)$, and San Diego $\left(P_{\mathrm{c}}=5.45 \mathrm{GV}\right)$. Line: ratio of Dorman functions shown in Figure 5(a),(b). Each dot represents a 1-hr data point.

\section{Response Functions}

To quantify the dependence shown in Figure 4 we performed a fit to a Dorman function [6] to the data for 3BC and 3NM64 separately. To do the fit we minimize the least squares function using the Levenburg-Marquardt algorithm, and provide estimates for the statistical errors in the determination of the parameters from the scatter of the data points about the resulting fit. The results of the fits are shown in Figure 5. In this case, as in subsequent ones, we show the best fit as a solid line and the (two sigma) range of possible fits as a shaded area.

\section{Conclusions}

We report an analysis of data taken using three bare neutron counters with a novel design and a standard three-counter neutron monitor (3NM64) on board the U.S. Coast Guard icebreaker Polar Star from 6 November 1995 to 20 March 1996, over a route from San Diego, USA to McMurdo Base, Antarctica and back to the northern Pacific Ocean. This latitude survey sampled geomagnetic cutoffs over $0<P_{\mathrm{c}}<15 \mathrm{GV}$, allowing a determination of the BC and 3NM64 response functions over that range. Various techniques were used to clean and correct the data. The pressure correction coefficient as a function of $P_{\mathrm{c}}$ was previously determined for this ship-borne 3NM64 (using data from 13 latitude surveys over 1994-2007 [9], and here we investigated whether the BC count rate 

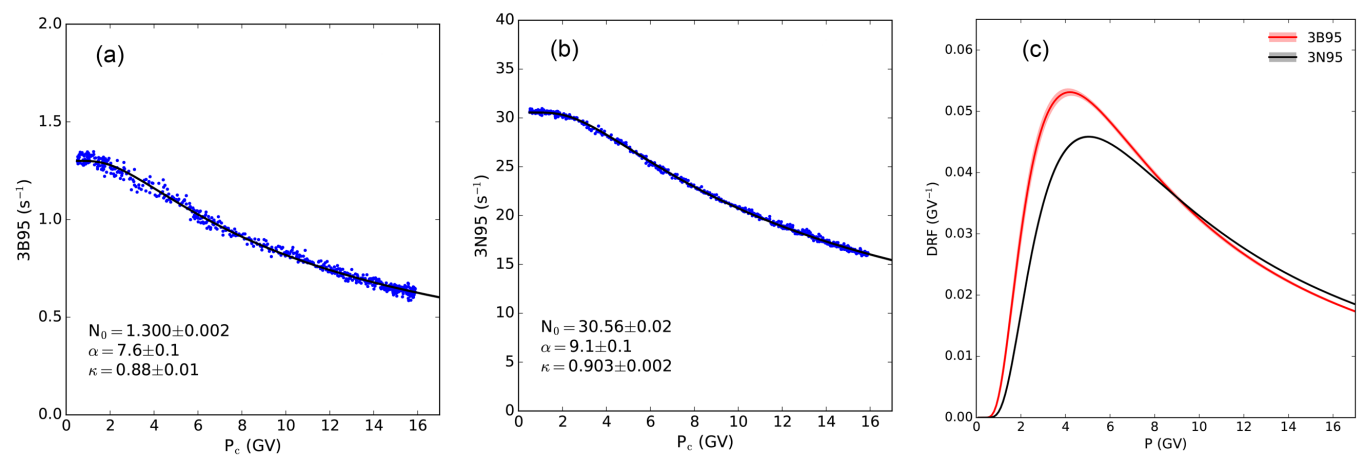

Figure 5: Dorman function fits to (a) bare and (b) neutron monitor data separately. (c) Comparison of the normalized differential response functions (bare neutron detectors and standard 3NM64) for the 1995 latitude survey. (Modified from [10].)

requires a different pressure coefficient. We concluded that the statistics available for these BCs are insufficient to determine whether the pressure coefficients are different, and therefore we used the same coefficient. The $\mathrm{BC}$ response was strongly affected by proximity to any port along the route, and such time periods were excluded when determining the response functions.

\section{Acknowledgments}

This study is supported in part by the Thailand Research Fund via grant RTA5980003 and Research Grant for New Scholar MRG6280155; by the U.S.National Science Foundation via awards PLR-1341562, PLR-1245939, and their predecessors; and by the Australian Antarctic Division.

\section{References}

[1] Bieber, J. W., \& Evenson, P. 1991, in Proc. 22nd ICRC (Dublin), 3, 129-132

[2] Bieber, J. W., Clem, J., \& Evenson, P. 1997, in Proc. 25th ICRC (Durban), 2, 389-392

[3] Bieber, J. W., Clem, J., Evenson, P., et al. 2013, ApJ, 771, 92

[4] Caballero-Lopez, R. A., \& Moraal, H. 2012, JGR, 117, A12, 103

[5] Clem, J. M., Bieber, J. W., Evenson, P., et al. 1997, JGR, 102, 26, 919-26

[6] Dorman, L. I., Fedchenko, S. G., et al. 1969, in Proc. 11th ICRC (Budapest), 2, 233-236

[7] Kouzes, R. T., E. R. Siciliano, J. H. Ely, et al. 2008, Nucl. Instrum. Meth. Phys. Res. A, 584, 383-400

[8] Hatton, C. J., \& Carmichael, H. 1964, Can. J. Phys., 42, 2443-2472

[9] Nuntiyakul, W., Evenson, P., Ruffolo D., et al. 2014, ApJ, 795, 11

[10] Nuntiyakul, W., Sáiz, A., Ruffolo, D., et al. 2018, JGR, 123, 7181

[11] Ruffolo, D., Sáiz, A., Mangeard, P.-S., et al. 2016, ApJ, 817, 38

[12] Simpson, J. A. Jr. 1948, PhRvL, 73, 1389-1391

[13] Tsyganenko, N. A. 1987, Planet. Space Sci., 35, 1347-1358

[14] Villoresi, G., Dorman, L. I., Iucci, N., et al. 2000, JGR, 105, 21, 025-21, 034

[15] Zreda, M., D. Desilets, T. P. A. Ferré, and R. L. Scott 2008, Geophys. Res. Lett., 35, L21,402 